\title{
On an equation of Goormaghtigh
}

\author{
by \\ Yu. V. Nesterenko (Moscow) and T. N. Shorey (Mumbai) \\ Dedicated to Professor A. Schinzel on his 60th birthday
}

1. Introduction. An equation of Goormaghtigh [4] is as follows:

(1) $\frac{x^{m}-1}{x-1}=\frac{y^{n}-1}{y-1} \quad$ in integers $x>1, y>1, m>2, n>2$ with $x \neq y$.

This equation asks for integers with all the digits equal to one with respect to two distinct bases. It has been conjectured that equation (1) has only finitely many solutions. Goormaghtigh [4] observed that

$$
31=\frac{2^{5}-1}{2-1}=\frac{5^{3}-1}{5-1}, \quad 8191=\frac{2^{13}-1}{2-1}=\frac{90^{3}-1}{90-1} .
$$

Nagell [5] confirmed a conjecture of Ramanujan [6] that the solutions of equation

$$
x^{2}+7=2^{n} \quad \text { in integers } x>0, n>0
$$

are given by $(x, n)=(1,3),(3,4),(5,5),(11,7),(181,15)$. This result implies that the solutions of equation (1) with $x=2$ and $n=3$ are given by (2). Perhaps equation (1) has no solution other than the ones given by (2). Shorey [10] proved that 31 and 8191 are the only primes $N$ such that $N$ has all the digits equal to 1 with respect to two distinct bases and $\omega(N-1) \leq 5$. Here $\omega(N-1)$ denotes the number of distinct prime divisors of $N-1$.

We re-write equation (1) as

$$
(y-1) x^{m}-(x-1) y^{n}=y-x .
$$

If $x$ and $y$ are fixed, we read the exponents $m$ and $n \bmod 3$ to write (3) as Thue equations with fixed coefficients and hence equation (1) has only finitely many solutions. Balasubramanian and Shorey [2] extended this result by showing that equation (1) implies that $\max (x, y, m, n)$ is bounded by an effectively computable number $C_{1}$ depending only on the greatest prime

1991 Mathematics Subject Classification: Primary 11D61. 
factor of $x$ and $y$. If $\operatorname{gcd}(x, y)=1$, Shorey [9] showed that we can replace $C_{1}$ in the above result by an effectively computable number $C_{2}$ depending only on the greatest prime factor of $x$ and $y-x$. The proofs of the preceding two results depend on the theory of linear forms in logarithms.

If $m$ and $n$ are fixed, Davenport, Lewis and Schinzel [3] proved that equation (1) has only finitely many solutions. They proved that the curve

$$
\frac{X^{m}-1}{X-1}-\frac{Y^{n}-1}{Y-1}=0
$$

is absolutely irreducible with positive genus and hence the assertion follows from a theorem of Siegel [12]. Since Siegel's result is not effective, it is not possible to give an effective bound for the magnitude of the solutions. If $\operatorname{gcd}(m-1, n-1)>1$, Davenport, Lewis and Schinzel replaced Siegel's result by Runge's result [7] which is effective. Hence they [3] proved effectively that equation (1) has only finitely many solutions whenever $m$ and $n$ are fixed such that $\operatorname{gcd}(m-1, n-1)>1$. The latter assumption is satisfied whenever $m$ and $n$ are odd. We extend this result as follows.

Theorem 1. Assume

$$
m-1=d r, \quad n-1=d s
$$

where $d, r$ and $s$ are positive integers satisfying

$$
d \geq 2, \quad \operatorname{gcd}(r, s)=1 .
$$

Then equation (1) implies that $\max (x, y, m, n)$ is bounded by an effectively computable number depending only on $r$ and $s$.

If $m$ and $n$ with $\operatorname{gcd}(m-1, n-1)>1$ are fixed, the assumptions (5) and (6) are satisfied with $\operatorname{gcd}(m-1, n-1)=d, r=(m-1) / d$ and $s=(n-1) / d$ and hence the above mentioned effective result of Davenport, Lewis and Schinzel follows from Theorem 1. Our proof is different in the sense that we do not require to show that curve (4) is irreducible essential in the proof of Davenport, Lewis and Schinzel. For given pairs $r, s$ of relatively prime positive integers, let $S_{r, s}$ be the set of all pairs $(m, n)=(1+d r, 1+d s)$ with $d=2,3, \ldots$ This is an infinite set and Theorem 1 implies that equation (1) has only finitely many solutions when the exponents $(m, n)$ are restricted to the set $S_{r, s}$. This is the first result of the type where there is no restriction (like the ones mentioned in the preceding paragraph) on the pairs $(x, y)$ and the pairs $(m, n)$ extend over an infinite set.

There is no loss of generality in assuming that $x<y$ in equation (1). Then $m>n$ and $r>s$. Theorem 1 is a consequence of the following result.

THEOREM 2. Suppose that equation (1) with $x<y$, (5) and (6) is satisfied. Then

$$
r \geq C_{3} m^{1 / 5}(\log m)^{-3 / 5}
$$

where $C_{3}>0$ is an effectively computable absolute constant. 
We apply the arguments of Saradha and Shorey [8] for obtaining the following explicit and sharp estimate for $y$ in terms of $d, r, s$ and we combine it with the theory of linear forms in logarithms to conclude the proof of Theorem 2 .

Theorem 3. Let $g=(d+1) / d^{2}$ and $D_{r}=d^{r} \prod_{p \mid d} p^{\text {ord }_{p}(r !)}$. Equation (1) with $x<y$, (5) and (6) implies that

$$
x<\max \left(9, \frac{g D_{r}}{2}+1\right) .
$$

By equation (1) and (5), we estimate

$$
y \leq 2 x^{r / s}
$$

which, together with (7), gives a bound for $y$ in terms of $d, r$ and $s$. Estimate (7) can be used to compute all the solutions of equation (1) for small values of $d, r$ and $s$. For example, we have that

Theorem 4. Equation (1) with $x<y, m \equiv 1(\bmod 2)$ and $n=3$ implies

$$
m \geq 25
$$

unless

$$
(x, y, m)=(2,5,5) \text { or }(2,90,13) .
$$

We used MATHEMATICA for the computations required for Theorem 4. We thank Dr. N. Saradha for writing a computer programme to carry out computations. We thank the referee for comments and remarks on an earlier draft of the paper. For an account of results on equation(1) and more general equation $a \frac{x^{m}-1}{x-1}=b \frac{y^{n}-1}{y-1}$, we refer to Shorey and Tijdeman [11, Chapter 12].

2. Preliminaries. We understand that an empty sum and an empty product is equal to 0 and 1 , respectively. Let $\mu \in\{m, n\}$ and $d>1$ be an integer given by (5) and (6). We put

$$
g=\frac{d+1}{d^{2}}, \quad t=\frac{\mu-1}{d} .
$$

We observe from (5) that

$$
t= \begin{cases}r & \text { if } \mu=m \\ s & \text { if } \mu=n\end{cases}
$$

Let $A_{\nu}(\mu, d)$ and $A_{\nu}(d)$ be rational numbers given by

$$
\left(1+\frac{1}{X}+\ldots+\frac{1}{X^{\mu-1}}\right)^{1 / d}=\sum_{\nu=0}^{\infty} \frac{A_{\nu}(\mu, d)}{X^{\nu}}
$$

and

$$
\left(1-\frac{1}{X}\right)^{-1 / d}=\sum_{\nu=0}^{\infty} \frac{A_{\nu}(d)}{X^{\nu}} \text {. }
$$


We observe that

$$
\begin{gathered}
A_{\nu}(d)=\frac{d^{-1}\left(d^{-1}+1\right) \ldots\left(d^{-1}+\nu-1\right)}{\nu !} \quad \text { for } \nu \geq 0, \\
A_{\nu}(d)=A_{\nu}(\mu, d) \quad \text { for } 0 \leq \nu<\mu .
\end{gathered}
$$

We derive that $A_{0}(d)=1, A_{1}(d)=1 / d$ and

$$
g / \nu \leq A_{\nu}(d) \leq g / 2 \quad \text { for } \nu \geq 2 .
$$

We put

$$
D_{\nu}=D_{\nu}(d)=d^{\nu} \prod_{p \mid d} p^{\operatorname{ord}_{p}(\nu !)} \quad \text { for } \nu \geq 0
$$

where the product is taken over primes. We have

$$
\operatorname{ord}_{p}(\nu !)=\left[\frac{\nu}{p}\right]+\left[\frac{\nu}{p^{2}}\right]+\ldots<\frac{\nu}{p-1} .
$$

By (15) and (16), we observe that

$$
D_{\nu} \leq d^{2 \nu} \quad \text { for } \nu \geq 0 .
$$

Further we observe from (13), (12) and (15) that

$$
D_{\nu} A_{\nu}(\mu, d) \in \mathbb{Z} \quad \text { for } 0 \leq \nu<\mu \text {. }
$$

\section{Proof of Theorems 3 and 4}

Proof of Theorem 3. Suppose that equation (1) with $9 \leq x<y$, (5) and

(6) is satisfied. Then $m>n$ and $r>s$. We re-write equation (1) as

$$
x^{m-1}\left(1+\frac{1}{x}+\ldots+\frac{1}{x^{m-1}}\right)=y^{n-1}\left(1+\frac{1}{y}+\ldots+\frac{1}{y^{n-1}}\right) .
$$

By taking $d$ th roots on both the sides of equation (19), we see from (5) and (10) that

$$
\begin{aligned}
& x^{r}\left(1+\frac{A_{1}(m, d)}{x}+\frac{A_{2}(m, d)}{x^{2}}+\ldots\right) \\
& =y^{s}\left(1+\frac{A_{1}(n, d)}{y}+\frac{A_{2}(n, d)}{y^{2}}+\ldots\right) .
\end{aligned}
$$

We put

and

$$
\begin{aligned}
\Lambda= & x^{r}+A_{1}(m, d) x^{r-1}+\ldots+A_{r}(m, d) \\
& -y^{s}-A_{1}(n, d) y^{s-1}-\ldots-A_{s}(n, d), \\
\Lambda_{1}= & \frac{A_{r+2}(m, d)}{x^{2}}+\frac{A_{r+3}(m, d)}{x^{3}}+\ldots, \\
\Lambda_{2}= & \frac{A_{r+1}(m, d)}{x}+\frac{A_{r+2}(m, d)}{x^{2}}+\ldots
\end{aligned}
$$

$$
\Lambda_{3}=\frac{A_{s+1}(n, d)}{y}+\frac{A_{s+2}(n, d)}{y^{2}}+\ldots
$$


First we show that $\Lambda_{1}, \Lambda_{2}$ and $\Lambda_{3}$ are positive. By (5) and (6), we observe that $r+1<m$. Then we see from (10), (13) with $\mu=m$ and (22) that

$$
x^{-r} \Lambda_{1}=\left(\frac{1-x^{-m}}{1-x^{-1}}\right)^{1 / d}-\sum_{\nu=0}^{r+1} \frac{A_{\nu}(d)}{x^{\nu}} .
$$

Therefore

$$
x^{-r} \Lambda_{1} \geq\left(1-\frac{2}{d} x^{-m}\right)\left(1-x^{-1}\right)^{-1 / d}-\sum_{\nu=0}^{r+1} \frac{A_{\nu}(d)}{x^{\nu}} .
$$

Now we use (26), (11) and (14) for deriving that

$$
\Lambda_{1} \geq \frac{A_{r+2}(d)}{x^{2}}\left(1-\frac{2(r+2)\left(1-x^{-1}\right)^{-1 / d}}{x^{m-r-2}}\right) .
$$

By (5), (6) and (14), we see that $m-r-2 \geq r-1$ and $A_{r+2}(d)>0$ which, together with $x \geq 9$, imply that $\Lambda_{1}>0$. By (23), (22), (13) with $\mu=m$ and (14), we derive that $\Lambda_{2}=A_{r+1}(d) / x+\Lambda_{1}>0$. The proof of $\Lambda_{3}>0$ is similar to that of $\Lambda_{1}>0$.

Now we give upper bounds for $\Lambda_{2}$ and $\Lambda_{3}$. As in (25), we have

$$
x^{-r} \Lambda_{2}=\left(\frac{1-x^{-m}}{1-x^{-1}}\right)^{1 / d}-\sum_{\nu=0}^{r} \frac{A_{\nu}(d)}{x^{\nu}} .
$$

Therefore we observe from (11) that

$$
\Lambda_{2}<\frac{A_{r+1}(d)}{x}+\frac{A_{r+2}(d)}{x^{2}}+\ldots
$$

By (12), we have

$$
\frac{A_{r+j}(d)}{A_{r+1}(d)}=\frac{\left(d^{-1}+r+1\right) \ldots\left(d^{-1}+r+j-1\right)}{(r+2) \ldots(r+j)} \leq 1 \quad \text { for } j=1,2, \ldots
$$

Consequently,

$$
\Lambda_{2}<\frac{A_{r+1}(d)}{x}\left(1+\frac{1}{x}+\ldots\right)=\frac{A_{r+1}(d)}{x-1} .
$$

Hence we apply (14) to conclude

$$
0<\Lambda_{2}<\frac{A_{r+1}(d)}{x-1} \leq \frac{g}{2(x-1)}
$$

Similarly we have

$$
0<\Lambda_{3}<\frac{A_{s+1}(d)}{y-1} \leq \frac{g}{2(y-1)} .
$$


We assume that $\Lambda \neq 0$. Then we observe from (21), (18), (15) and $r>s$ that $D_{r} \Lambda$ is a non-zero integer. Therefore

$$
|\Lambda| \geq D_{r}^{-1} .
$$

By (21), (20), (24) and (23), we have $\Lambda=\Lambda_{3}-\Lambda_{2}$. Now we apply (27), (28) and $x<y$ for deriving that

$$
|\Lambda|<\max \left(\Lambda_{3}, \Lambda_{2}\right)<\frac{g}{2(x-1)} .
$$

By combining (29) and (30), we conclude that $x<g D_{r} / 2+1$ and the assertion (7) follows. Therefore we may suppose that

$$
\Lambda=0 .
$$

By combining (20), (31), (22), (24) and (13), we find that $A_{r+1}(d)=$ $x\left(\Lambda_{3}-\Lambda_{1}\right)$. Now we use $\Lambda_{1}>0$ and (28) for deriving that

$$
A_{r+1}(d)<x \Lambda_{3}<\frac{x}{y-1} A_{s+1}(d) .
$$

By (12), we have

$$
\frac{A_{s+1}(d)}{A_{r+1}(d)}=\frac{(s+2) \ldots(r+1)}{\left(d^{-1}+s+1\right) \ldots\left(d^{-1}+r\right)}<\frac{r+1}{s+1} .
$$

Thus

$$
y-1<\frac{r+1}{s+1} x
$$

since $A_{r+1}(d)>0$ by (14). By equation (1), we have

$$
x^{m-1}<\frac{y^{n}}{y-1}=\left(1+\frac{1}{y-1}\right)^{n}(y-1)^{n-1} .
$$

Now we see from (5) and $y-1 \geq x$ that

$$
x^{r / s}<\left(1+\frac{1}{y-1}\right)^{n /(n-1)}(y-1)<\left(1+\frac{1}{x}\right)^{n /(n-1)} \frac{r+1}{s+1} x .
$$

Therefore

$$
x<\left(1+\frac{1}{x}\right)^{\frac{n}{n-1} \cdot \frac{s}{r-s}}\left(\frac{r+1}{s+1}\right)^{s /(r-s)} .
$$

Further we observe that

$$
\left(\frac{r+1}{s+1}\right)^{s /(r-s)}=\left(1+\frac{r-s}{s+1}\right)^{s /(r-s)}<e^{\frac{r-s}{s+1} \cdot \frac{s}{r-s}}<e
$$

and

$$
\left(1+\frac{1}{x}\right)^{\frac{n}{n-1} \cdot \frac{s}{r-s}}<e^{\frac{3}{2 x} \cdot \frac{s}{r-s}}
$$


since $n \geq 3$. Consequently, we have

$$
x<e^{1+\frac{3}{2 x} \cdot \frac{s}{r-s}} .
$$

On the other hand, $x \geq 9>e^{2}$. Hence $e^{2}<e^{1+\frac{3}{2 x} \cdot \frac{s}{r-s}}$. Thus

$$
x<\frac{3}{2} \cdot \frac{s}{r-s} \leq \frac{3}{2}(r-1) .
$$

Further we observe from (15) and (9) that

Hence $x<g D_{r} / 2+1$.

$$
\frac{g D_{r}}{2} \geq \frac{d+1}{2} d^{r-2} \geq \frac{3}{2} 2^{r-2} \geq \frac{3}{2}(r-1) .
$$

Proof of Theorem 4. Suppose that equation (1) with $x<y, m \equiv 1$ $(\bmod 2), m \leq 23$ and $n=3$ is satisfied. Then (5) and (6) are valid with $d=2, s=1$ and $1<r \leq 11$. Further $g=3 / 4$ by $(9)$ and $D_{r}=2^{r+\operatorname{ord}_{2}(r !)}$ by (15). Now we conclude (7) from Theorem 3 . We re-write equation (1) as

$$
4\left(\frac{x^{m}-1}{x-1}\right)-3=(2 y+1)^{2}
$$

and

$$
x \frac{x^{m-1}-1}{x-1}=y(y+1) .
$$

Let $m=17$. If $x \equiv 1(\bmod 3)$, we observe that the left hand side of (33) is $\equiv 1(\bmod 3)$. On the other hand, the right hand side of $(33)$ is $\equiv 0,2$ $(\bmod 3)$. If $x \equiv 3,4,5(\bmod 7)$, we see that the left hand side of (33) is $\equiv 1,4,3(\bmod 7)$, respectively, whereas the right hand side of (33) is always $\equiv 0,2,5,6(\bmod 7)$. Hence

$$
x \not \equiv 1(\bmod 3), \quad x \not \equiv 3,4,5(\bmod 7) \quad \text { if } m=17 .
$$

Similarly we derive that

$$
x \not \equiv 1(\bmod 5), \quad x \not \equiv 1(\bmod 7) \quad \text { if } m=19
$$

and

$$
x \not \equiv 1(\bmod 3), \quad x \not \equiv 1,3,4,5(\bmod 7) \quad \text { if } m=23 .
$$

For every $x$ satisfying (7), (34), (35) and (36), we check on a computer that the square root of the left hand side of (32) is not an integer unless $(x, y, m)=(2,5,5)$ or $(2,90,13)$.

4. Linear forms in logarithms. As mentioned in Section 1, the proof of Theorem 2 depends on the theory of linear forms in logarithms. The height of a non-zero rational number is the maximum of the absolute values of its numerator and denominator. We state the following estimate of Baker and Wüstholz [1] on linear forms in logarithms. 
TheOREM A. Let $n>1$ and $\alpha_{1}, \ldots, \alpha_{n}$ be positive rational numbers of heights not exceeding $A_{1}, \ldots, A_{n}$, respectively, where $A_{j} \geq e$ for $1 \leq j \leq n$. Put

$$
\Omega=\prod_{j=1}^{n} \log A_{j}
$$

Then the inequalities

$$
0<\left|b_{1} \log \alpha_{1}+\ldots+b_{n} \log \alpha_{n}\right|<\exp \left(-(16 n)^{2(n+2)} \Omega \log B\right)
$$

have no solution in integers $b_{1}, \ldots, b_{n}$ of absolute values not exceeding $B$ where $B \geq e$.

5. Proof of Theorems 1 and 2. Suppose that equation (1) with (5) and (6) is satisfied.

Proof of Theorem 2. We denote by $c_{1}, \ldots, c_{5}$ effectively computable absolute positive constants. Let $x<y$. Then $m>n$ and $r>s$. Further we re-write equation (1) as

$$
\frac{x^{m}}{x-1}-\frac{y^{n}}{y-1}=\frac{1}{x-1}-\frac{1}{y-1} .
$$

Thus

which implies that

$$
0<\left|y^{n} x^{-m}\left(\frac{x-1}{y-1}\right)-1\right|<x^{-m}
$$

$$
0<\left|n \log y-m \log x+\log \left(\frac{x-1}{y-1}\right)\right|<2 x^{-m} .
$$

Now we apply Theorem A with $A_{1}=A_{2}=A_{3}=y$ and $B=m$ for deriving that

$$
\left|n \log y-m \log x+\log \left(\frac{x-1}{y-1}\right)\right| \geq \exp \left(-c_{1}(\log y)^{3} \log m\right) .
$$

Thus

$$
m \leq c_{2}(\log y)^{3}(\log m) /(\log x)
$$

which, together with (8), implies that

$$
m / r \leq m s / r \leq c_{3}(\log y)^{2}(\log m) .
$$

By (8), (7), (17) and $d<m$, we estimate

$$
y \leq 2 x^{r / s} \leq d^{c_{4} r^{2} / s}<m^{c_{4} r^{2} / s} .
$$

Finally we combine (38) and (37) for deriving that

$$
r^{2} \geq c_{5} \frac{\log y}{\log m} \geq c_{6}\left(\frac{m}{r(\log m)^{3}}\right)^{1 / 2}
$$


implying that

$$
r \geq c_{7} m^{1 / 5}(\log m)^{-3 / 5}
$$

Proof of Theorem 1. There is no loss of generality in assuming that $x<y$ implying that $m>n$ and $r>s$. First we apply Theorem 2 to conclude that $m$ and $n$ are bounded by an effectively computable number depending only on $r$. Then we conclude from (7) and (8) that $x$ and $y$ are bounded by an effectively computable number depending only on $r$.

\section{References}

[1] A. Baker and G. Wüstholz, Logarithmic forms and group varieties, J. Reine Angew. Math. 442 (1993), 19-62.

[2] R. Balasubramanian and T. N. Shorey, On the equation $a\left(x^{m}-1\right) /(x-1)=$ $b\left(y^{n}-1\right) /(y-1)$, Math. Scand. 46 (1980), 177-182.

[3] H. Davenport, D. J. Lew is and A. Schinzel, Equations of the form $f(x)=g(y)$, Quart. J. Math. Oxford Ser. (2) 12 (1961), 304-312.

[4] R. Goormaghtigh, L'Intermédiaire des Mathématiciens 24 (1917), 88.

[5] T. Nagell, The diophantine equation $x^{2}+7=2^{n}$, Ark. Mat. 4 (1961), 185-187.

[6] S. Ramanujan, Question 464, J. Indian Math. Soc. 5 (1913), Collected Papers, Cambridge Univ. Press, 1927, 327.

[7] C. Runge, Ueber ganzzahlige Los̈ungen von Gleichungen zwischen zwei Veränderlichen, J. Reine Angew. Math. 100 (1887), 425-435.

[8] N. Saradha and T. N. Shorey, On the equation $(x+1) \ldots(x+k)=(y+1) \ldots(y+$ $m k$ ), Indag. Math. (N.S.) 3 (1992), 79-90.

[9] T. N. Shorey, On the equation $a\left(x^{m}-1\right) /(x-1)=b\left(y^{n}-1\right) /(y-1)($ II $)$, HardyRamanujan J. 7 (1984), 1-10.

[10] - Integers with identical digits, Acta Arith. 53 (1989), 187-205.

[11] T. N. Shorey and R. Tijdeman, Exponential Diophantine Equations, Cambridge Tracts in Math. 87, Cambridge Univ. Press, 1986.

[12] C. L. Siegel, Ueber einige Anwendungen diophantischer Approximationen, Abh Preuss. Akad. Wiss. Phys.-Math. Kl. 1 (1929), 70 pp.

Department of Mathematics

Moscow State University

Moscow 119899, Russia

E-mail: nest@nw.math.msu.su
School of Mathematics Tata Institute of Fundamental Research Homi Bhabha Road Mumbai 400 005, India E-mail: shorey@math.tifr.res.in 